\title{
Top quark and Higgs boson masses in supersymmetric models
}

\section{Ilia Gogoladze, ${ }^{a, 1}$ Rizwan Khalid, $^{b}$ Shabbar Raza ${ }^{c}$ and Qaisar Shafi ${ }^{a}$}

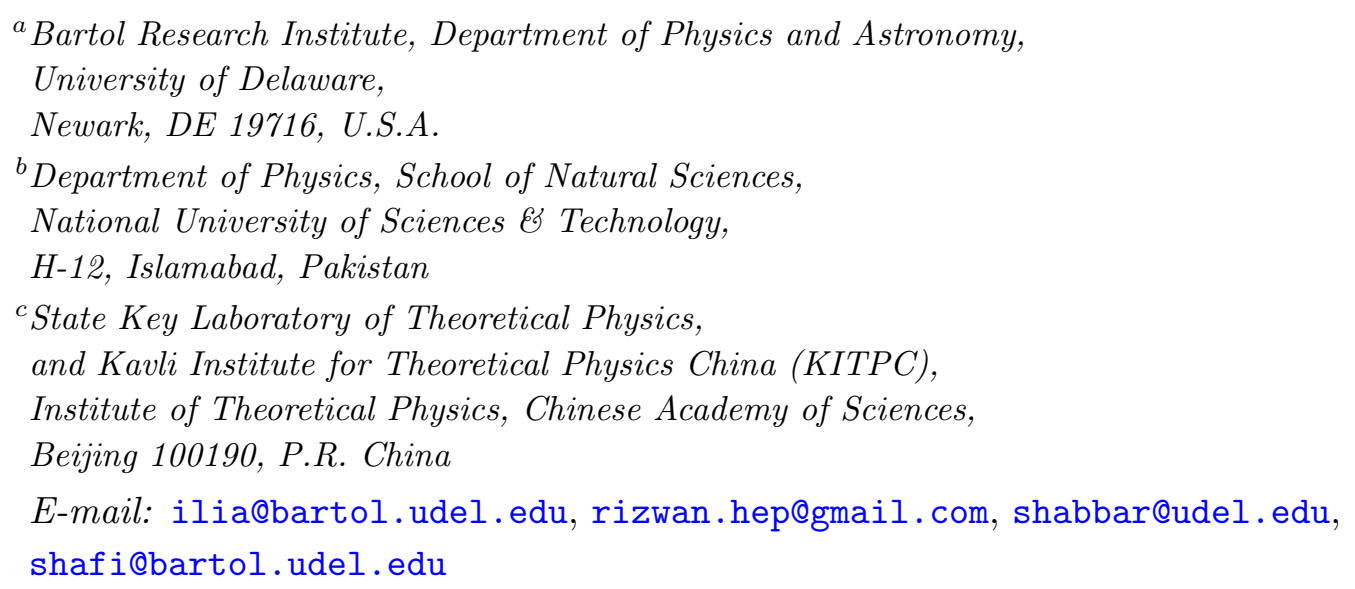

ABSTRACT: We study the implications for bounds on the top quark pole mass $m_{t}$ in models with low scale supersymmetry following the discovery of the Standard Model-like Higgs boson. In the minimal supersymmetric standard model, we find that $m_{t} \geq 164 \mathrm{GeV}$, if the light $\mathrm{CP}$ even Higgs boson mass $m_{h}=125 \pm 2 \mathrm{GeV}$. We also explore the top quark and Higgs boson masses in two classes of supersymmetric $\mathrm{SO}(10)$ models with t-b- $\tau$ Yukawa coupling unification at $M_{\mathrm{GUT}}$. In particular, assuming $\mathrm{SO}(10)$ compatible non-universal gaugino masses, setting $m_{h}=125 \mathrm{GeV}$ and requiring $5 \%$ or better Yukawa unification, we obtain the result $172 \mathrm{GeV} \leq m_{t} \leq 175 \mathrm{GeV}$. Conversely, demanding $5 \%$ or better $t-b-\tau$ Yukawa unification and setting $m_{t}=173.2 \mathrm{GeV}$, the Higgs boson mass is predicted to lie in the range $122 \mathrm{GeV} \leq m_{h} \leq 126 \mathrm{GeV}$.

KEYwORDS: Supersymmetry Phenomenology

ARXIV EPRINT: 1402.2924

\footnotetext{
${ }^{1}$ On leave of absence from: Andronikashvili Institute of Physics, 0177 Tbilisi, Georgia.
} 


\section{Contents}

1 Introduction 1

2 Phenomenological constraints and scanning procedure 3

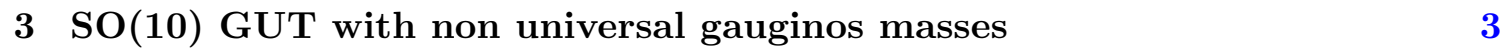

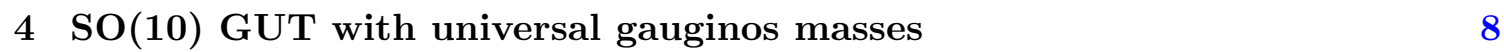

$\begin{array}{lll}5 & \text { Conclusions } & 11\end{array}$

\section{Introduction}

With the discovery by the ATLAS [1] and CMS [2] collaborations of a Standard Model (SM)-like Higgs boson with mass $m_{h} \simeq 125-126 \mathrm{GeV}$, the particle content of the $\mathrm{SM}$ is fully verified, and the top quark with mass $m_{t}=173.18 \pm 0.94 \mathrm{GeV}[3]$ remains the heaviest particle. The flavor structure of the SM, and, in particular, the relatively heavy top quark mass compared with other fermion masses is an open question in the SM. Likewise, as far as the SM is concerned, there is no deep reason for the Higgs boson to have mass $m_{h} \simeq 125-126 \mathrm{GeV}$.

At the same time, one of the successes of the SM is a relatively accurate prediction of the top quark pole mass $m_{t}$ from fits to the electroweak data. The top quark 'prediction' deduced from such fits does not change much as a result of the Higgs boson discovery due to a relatively mild dependence on the latter [4-7]. This mild dependence of the electroweak fits on the Higgs boson mass can be understood from the one loop radiative corrections to the $W$-boson mass. While these corrections depend quadratically on the top quark mass, there is only a mild logarithmic dependence on the SM Higgs boson mass. Employing all experimental data (including $m_{h}=125.7 \mathrm{GeV}$ ), fits to the electroweak data at the $95 \%$ confidence level yield $168 \mathrm{GeV} \lesssim m_{t} \lesssim 178 \mathrm{GeV}$, and $80.3 \mathrm{GeV} \lesssim M_{W} \lesssim 80.4 \mathrm{GeV}$ [4] for the $W$-boson mass, in good agreement with the direct measurement of these quantities. Note that while it is possible to constrain the top quark in the SM from rare decays, such constraints are not compatible with the bounds obtained from the determination of the $W$ boson mass [4-7].

Supersymmetry remains a compelling extensions of the SM and the top quark plays a key role in several features of the minimal supersymmetric standard model (MSSM). For instance, the top quark Yukawa coupling is the dominant contributor to the mechanism of radiative electroweak symmetry breaking (REWSB). We discuss in this paper how the REWSB condition restricts the top quark mass as a function of $\tan \beta$, the ratio of the vacuum expectation values of the two MSSM Higgs doublets. The top quark also plays 
a crucial role in the calculation of radiative corrections to the lightest $\mathrm{CP}$ even Higgs boson mass in the the MSSM. Among other things these corrections, to leading order, are proportional to the fourth power of the top quark mass. In addition, there is a logarithmic dependence on the geometric mean $M_{S}$ of the stop quark masses, as well as a contribution proportional to $\left(A_{t} / M_{S}\right)^{4}$, where $A_{t}$ is a tri-linear soft supersymmetry breaking (SSB) term [8-13]. We show in this paper that a $125 \mathrm{GeV}$ SM-like Higgs boson provides strong constraints on the allowed mass range for the top quark mass. This mass interval turns out to be compatible with the range obtained from fits to the electroweak data.

In a supersymmetric $\mathrm{SO}(10)$ grand unified theory (GUT) with $t-b-\tau$ Yukawa unification [14-16], it was shown in [17, 18] that with suitable non-universal SSB gaugino masses at $M_{\mathrm{GUT}}$ the lightest CP even Higgs boson mass is predicted to lie close to $125 \mathrm{GeV}$. Such gaugino non-universality at $M_{\mathrm{GUT}}$ can arise, for instance, from a non-singlet $F$-component of the field that breaks supersymmetry. Motivated by this result we seek to provide an answer in this paper to the following question: can models compatible with $t$-b- $\tau$ Yukawa unification also yield a stringent constraint for the top quark mass in good agreement with the observations?

We consider two classes of $\mathrm{SO}(10)$ models with a minimal set of SSB parameters at $M_{\mathrm{GUT}}$ in which $t-b-\tau$ Yukawa unification is realized. The most well studied one has universal SSB gaugino mass terms but non-universal Higgs SSB terms, $m_{H_{u}}^{2} \neq m_{H_{d}}^{2}$ at $M_{\mathrm{GUT}}$. Here $m_{H_{u}, H_{d}}^{2}$ stand for the up/down type Higgs SSB masses ${ }^{2}$. The second class of models, mentioned earlier, assumes universal Higgs SSB mass ${ }^{2}$ terms, but the gaugino SSB masses at $M_{\mathrm{GUT}}$ are non-universal. Allowing the top quark mass to vary in the interval $0<m_{t}<220 \mathrm{GeV}$, we scan the characteristic SSB parameter space for both classes of models and show that the $t-b-\tau$ Yukawa unification condition yields to a relatively narrow interval for the masses of the top quark and the light CP even Higgs boson. An upper bound for the top quark mass is obtained by imposing perturbativity on the top Yukawa coupling up to $M_{\mathrm{GUT}}$. It is possible [19-28] to relax the universality of SSB Higgs mass ${ }^{2}$ terms along with the non-universality of SSB gaugino mass terms, but we restrict ourselves here to the above two classes.

The outline for the rest of the paper is as follows. In section 2 we summarize the scanning procedure and the experimental constraints applied in our analysis. In section 3 we present the results for the $\mathrm{SO}(10)$ model in which $t-b-\tau$ Yukawa unification is achieved via non-universal SSB gaugino masses and universal up and down Higgs SSB mass ${ }^{2}$ terms $\left(m_{H_{u}}^{2}=m_{H_{d}}^{2}=m_{10}^{2}\right)$. Using the fact that the light $\mathrm{CP}$ even Higgs boson mass is in the interval $123 \mathrm{GeV}<m_{h}<127 \mathrm{GeV}$, we show that the top quark mass lies in the range $164 \mathrm{GeV}<m_{t}<205 \mathrm{GeV}$. Imposing $5 \%$ or better $t-b-\tau$ Yukawa unification, the allowed top quark mass range shrinks to $168 \mathrm{GeV}<m_{t}<180 \mathrm{GeV}$. In section 4 we present results for the $\mathrm{SO}(10)$ model with universal SSB gaugino mass terms but which also requires that $m_{H_{u}}^{2}<m_{H_{d}}^{2}[29,30]$. In this case, we find the top quark mass range $165 \mathrm{GeV}<m_{t}<200 \mathrm{GeV}$. Imposing $5 \%$ or better $t$-b- $\tau$ Yukawa unification condition, the interval for the top quark mass is reduced to $170 \mathrm{GeV}<m_{t}<178 \mathrm{GeV}$. We also consider $b$ - $\tau$ Yukawa unification in this case and, as expected, the constraint on the top quark mass is relaxed to $168 \mathrm{GeV}<m_{t}<200 \mathrm{GeV}$. Our conclusions are presented in section 5 . 


\section{Phenomenological constraints and scanning procedure}

We employ the ISAJET 7.84 package [31] to perform random scans over the parameter space. In this package, the weak scale values of gauge and third generation Yukawa couplings are evolved to $M_{\mathrm{GUT}}$ via the MSSM renormalization group equations (RGEs) in the $\overline{D R}$ regularization scheme. We do not strictly enforce the unification condition $g_{3}=g_{1}=g_{2}$ at $M_{\mathrm{GUT}}$, since a few percent deviation from unification can be assigned to unknown GUTscale threshold corrections [32-34]. With the boundary conditions given at $M_{\mathrm{GUT}}$, all the SSB parameters, along with the gauge and third family Yukawa couplings, are evolved back to the weak scale $M_{Z}$.

In evaluating the Yukawa couplings the SUSY threshold corrections [35] are taken into account at a common scale $M_{S}=\sqrt{m_{\tilde{t}_{L}} m_{\tilde{t}_{R}}}$. The entire parameter set is iteratively run between $M_{\mathrm{Z}}$ and $M_{\mathrm{GUT}}$ using the full 2-loop RGEs until a stable solution is obtained. To better account for the leading-log corrections, one-loop step-beta functions are adopted for the gauge and Yukawa couplings, and the SSB scalar mass parameters $m_{i}$ are extracted from RGEs at appropriate scales $m_{i}=m_{i}\left(m_{i}\right)$. The RGE-improved 1-loop effective potential is minimized at an optimized scale $M_{S}$, which effectively accounts for the leading 2-loop corrections. Full 1-loop radiative corrections are incorporated for all sparticle masses.

In scanning the parameter space, we employ the Metropolis-Hastings algorithm as described in [36]. The data points collected all satisfy the requirement of radiative electroweak symmetry breaking (REWSB) [37-41], with the neutralino in each case being the LSP. After collecting the data, we impose the mass bounds on all the particles [42] and use the IsaTools package [43, 44] and SuperIso v2.3 [45] to implement the following phenomenological constraints:

$$
\begin{array}{rlrl}
0.8 \times 10^{-9} & \leq B R\left(B_{s} \rightarrow \mu^{+} \mu^{-}\right) & & \leq 6.2 \times 10^{-9}(2 \sigma) \\
2.99 \times 10^{-4} & \leq B R(b \rightarrow s \gamma) & & \leq 3.87 \times 10^{-4}(2 \sigma) \\
0.15 & \leq \frac{B R\left(B_{u} \rightarrow \tau \nu_{\tau}\right)_{\mathrm{MSSM}}}{B R\left(B_{u} \rightarrow \tau \nu_{\tau}\right)_{\mathrm{SM}}} & \leq 2.41(3 \sigma) & {[47]} \\
0 & \leq \Delta(g-2)_{\mu} / 2 & \leq 55.6 \times 10^{-10}
\end{array}
$$

We also implement the following mass bounds on the sparticle masses:

$$
\begin{array}{lll}
m_{\tilde{g}} \gtrsim 1.4 \mathrm{TeV} & \left(\text { for } \quad m_{\tilde{g}} \sim m_{\tilde{q}}\right) & {[49,50]} \\
m_{\tilde{g}} \gtrsim 0.9 \mathrm{TeV} & \left(\text { for } \quad m_{\tilde{g}} \ll m_{\tilde{q}}\right) & {[49,50]} \\
M_{A} \gtrsim 700 \mathrm{GeV} & (\text { for } \tan \beta \simeq 48) & {[51] .}
\end{array}
$$

\section{SO(10) GUT with non universal gauginos masses}

One of the main motivations of supersymmetric $\mathrm{SO}(10) \mathrm{GUT}$, in addition to gauge coupling unification, is matter unification. The spinor representation of $\mathrm{SO}(10)$ unifies all matter fermions of a given family in a single multiplet $\left(16_{i}\right)$, which also contains the right handed neutrino $\left(\nu_{R}\right)$. Another virtue of $\mathrm{SO}(10)$ is that, in principle, the two MSSM Higgs doublets 
can be accommodated in a single ten dimensional $\left(10_{H}\right)$ representation, which then yields the following Yukawa couplings

$$
Y_{i j} 16_{i} 16_{j} 10_{\mathrm{H}} \text {. }
$$

Here $i, j=1,2,3$ stand for family indices and the $\mathrm{SO}(10)$ indices have been omitted for simplicity. For the third generation quarks and leptons, the interaction in eq. (3.1) yields the following Yukawa coupling unification condition at $M_{\mathrm{GUT}}$ [14-16]

$$
Y_{t}=Y_{b}=Y_{\tau}=Y_{\nu_{\tau}} .
$$

In gravity mediated supersymmetry breaking scenario [52-55], implementing eq. (3.2), in particular $Y_{t}=Y_{b}=Y_{\tau}$ at $M_{\mathrm{GUT}}$, can place significant constraints on the supersymmetric spectrum [56]. These constraints depend on the particular boundary conditions for sparticle SSB masses chosen at $M_{\mathrm{GUT}}$. If the SSB gaugino mass terms are assumed to be universal at $M_{\mathrm{GUT}}, m_{H_{u}}^{2}\left(M_{\mathrm{GUT}}\right)<m_{H_{d}}^{2}\left(M_{\mathrm{GUT}}\right)$ is required in order for Yukawa coupling unification to be consistent with radiative electroweak symmetry breaking (REWSB). This splitting may arise, for example, via a $D$-term contribution to all scalar masses, or it can be generated via "Just-So" splitting $[29,30]$. The results of this scenario are presented in section 4 .

Alternatively, it is possible to achieve $t-b-\tau$ Yukawa coupling unification consistent with REWSB by assuming the gaugino SSB mass terms to be non-universal at $M_{\mathrm{GUT}}$. In this case, non-universality among the Higgs SSB mass terms is not needed.

It has been pointed out [57-59] that non-universal MSSM gaugino masses at $M_{\mathrm{GUT}}$ can arise from non-singlet $F$-terms, compatible with the underlying GUT symmetry. The SSB gaugino masses in supergravity [52-55] can arise, say, from the following dimension five operator:

$$
-\frac{F^{a b}}{2 M_{\mathrm{P}}} \lambda^{a} \lambda^{b}+\text { c.c. . }
$$

Here $\lambda^{a}$ is the two-component gaugino field, $F^{a b}$ denotes the $F$-component of the field which breaks SUSY, and the indices $a, b$ run over the adjoint representation of the gauge group. The resulting gaugino mass matrix is $\left\langle F^{a b}\right\rangle / M_{\mathrm{P}}$, where the supersymmetry breaking parameter $\left\langle F^{a b}\right\rangle$ transforms as a singlet under the MSSM gauge group $\mathrm{SU}(3)_{c} \times \mathrm{SU}(2)_{L} \times$ $\mathrm{U}(1)_{Y}$.

If $F$ transforms as a 54 or 210 dimensional representation of $\mathrm{SO}(10)$ [57-59], one obtains the following relation among the MSSM gaugino masses at $M_{\mathrm{GUT}}$ :

$$
M_{3}: M_{2}: M_{1}=2:-3:-1,
$$

where $M_{1}, M_{2}, M_{3}$ denote the gaugino masses corresponding to $\mathrm{U}(1), \mathrm{SU}(2)_{L}$ and $\mathrm{SU}(3)_{c}$, respectively. In order to obtain the correct sign for the desired contribution to $(g-2)_{\mu}$, we choose $M_{1}>0, M_{2}>0$ and $M_{3}<0$. Notice that, in general, if $F^{a b}$ transforms non trivially under $\mathrm{SO}(10)$, the SSB terms such as the trilinear couplings and scalar mass terms are not necessarily universal at $M_{G U T}$. However, we can assume, consistent with $\mathrm{SO}(10)$ gauge symmetry, that the coefficients associated with terms that violate the $\mathrm{SO}(10)$-invariant form are suitably small, except for the gaugino term in eq. (3.3). We also assume that D-term contributions to the SSB terms are much smaller compared with contributions from fields with non-zero auxiliary $F$-terms. 
Employing the boundary condition from eq. (3.4), one can define the MSSM gaugino masses at $M_{\mathrm{GUT}}$ in terms of the mass parameter $M_{1 / 2}$ :

$$
\begin{aligned}
& M_{1}=M_{1 / 2} \\
& M_{2}=3 M_{1 / 2} \\
& M_{3}=-2 M_{1 / 2} .
\end{aligned}
$$

Note that $M_{2}$ and $M_{3}$ have opposite signs which is important in implementing Yukawa coupling unification as shown in [19-22]. In order to quantify Yukawa coupling unification, following [60], we define the quantity $R_{t b \tau}$ as,

$$
R_{t b \tau}=\frac{\max \left(Y_{t}, Y_{b}, Y_{\tau}\right)}{\min \left(Y_{t}, Y_{b}, Y_{\tau}\right)}
$$

We have performed random scans in the fundamental parameter space as follows:

$$
\begin{aligned}
0 & \leq m_{16} \leq 10 \mathrm{TeV} \\
0 & \leq M_{1 / 2} \leq 5 \mathrm{TeV} \\
0 & \leq m_{10} \leq 10 \mathrm{TeV} \\
-3 & \leq A_{0} / m_{16} \leq 2 \mathrm{TeV} \\
1.1 & \leq \tan \beta \leq 60 \\
0 & \leq m_{t} \leq 220 \mathrm{GeV} \\
\mu & >0 .
\end{aligned}
$$

Here $m_{16}$ is the universal SSB mass for MSSM sfermions, $m_{10}$ is the universal SSB mass term for the up/down MSSM Higgs doublets, $M_{1 / 2}$ is the gaugino mass parameter, $\tan \beta$ is the ratio of the vacuum expectation values (VEVs) of the two MSSM Higgs doublets, $A_{0}$ is the universal SSB trilinear scalar interaction (with corresponding Yukawa couplings factored out), $m_{t}$ denotes the top-quark mass and $\mu>0$ sets the sign for the bi-linear SSB Higgs mixing term whose absolute value is fixed by requiring REWSB.

In figure 1 , we present our results in the $m_{t}$ - $\tan \beta$ and $m_{t}-M_{S}$ planes. Gray points are consistent with REWSB and neutralino LSP. Blue points form a subset of the gray ones and satisfy sparticle mass bounds and other constraints described in section 2. Orange points belong to a subset of blue points and satisfy the lightest CP even Higgs boson mass bound $123 \mathrm{GeV} \leq m_{h} \leq 127 \mathrm{GeV}$.

It is interesting to note that REWSB can arise even for $m_{t}$ values as low as $10 \mathrm{GeV}$, but $\tan \beta$ values are then constrained to be in a narrow range. For a given top quark mass we have a well defined $\tan \beta$ interval from the requirement of REWSB. For instance, from the $m_{t}$-tan $\beta$ plane, one sees that in order to have REWSB with $m_{t} \approx 10 \mathrm{GeV}$, the value of $\tan \beta$ should be around 3 or so. Applying all the collider and B-physics constraints except the lightest Higgs boson mass bound, we obtain for the top quark mass the bound $125 \mathrm{GeV} \lesssim m_{t} \lesssim 208 \mathrm{GeV}$ (blue points in figure 1). Applying next the Higgs boson mass bound $123 \mathrm{GeV} \leq m_{h} \leq 127 \mathrm{GeV}$, the top quark is expected to lie in the interval $164 \mathrm{GeV} \lesssim m_{t} \lesssim 205 \mathrm{GeV}$. Similar observations can be made from the $m_{t}-M_{S}$ plane. It 

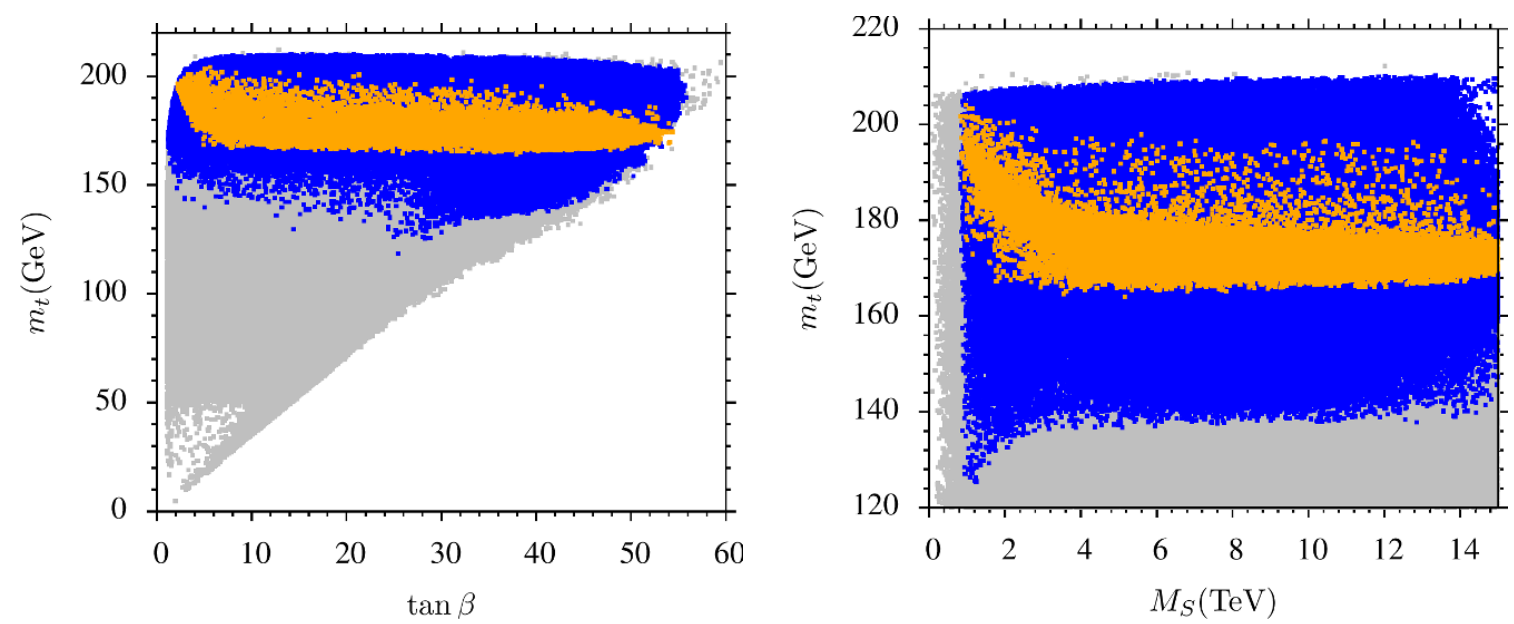

Figure 1. Plots in the $m_{t}$-tan $\beta$ and $m_{t}-M_{S}$ planes. Gray points are consistent with REWSB and neutralino LSP. Blue points form a subset of the gray and satisfy sparticle mass bounds and other constraints described in section 2. Orange points belong to a subset of blue points and satisfy the lightest $\mathrm{CP}$ even Higgs boson mass bound $123 \mathrm{GeV} \leq m_{h} \leq 127 \mathrm{GeV}$.
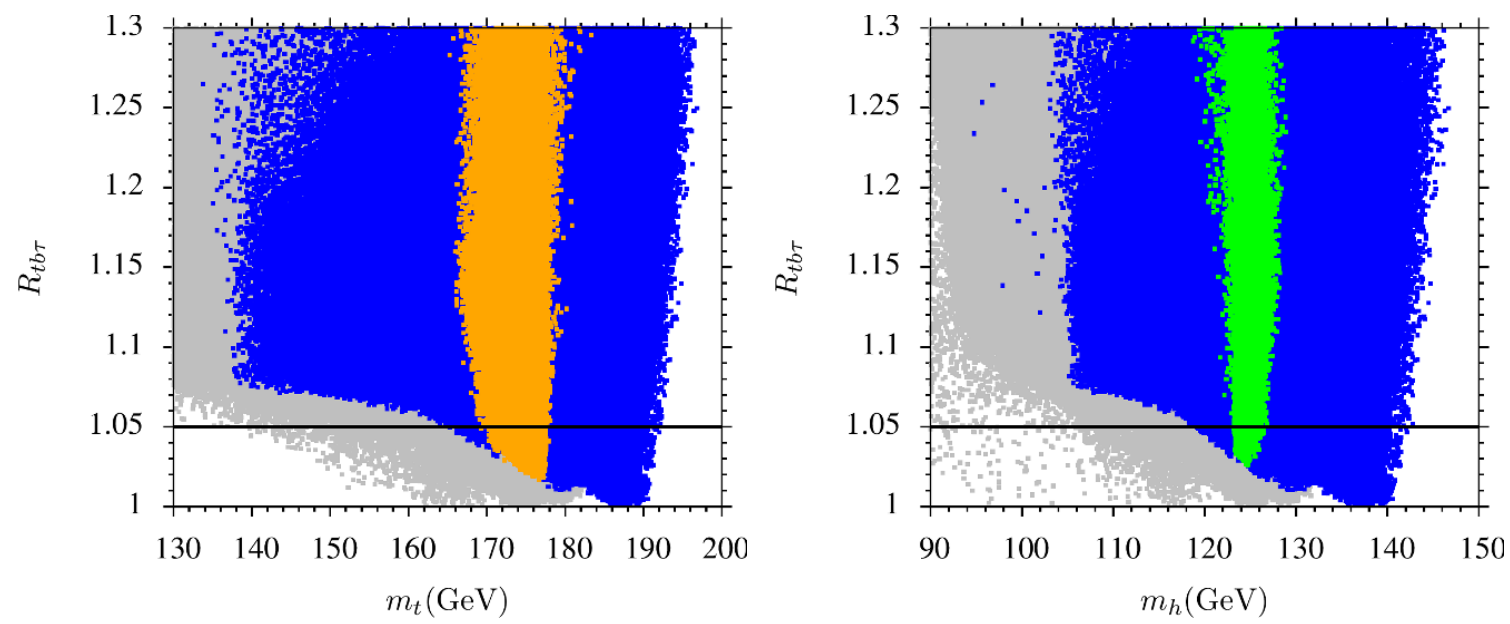

Figure 2. Plots in the $R_{t b \tau}-m_{t}$ and $R_{t b \tau}-m_{h}$ planes. Color coding is same as in figure 1. The green points in the $R_{t b \tau}-m_{h}$ plane form a subset of blue points and satisfy the bound $172.3 \mathrm{GeV} \leq m_{t} \leq$ $174.1 \mathrm{GeV}$.

is evident from this plane that no matter how heavy the stop mass, the top quark cannot be lighter than $164 \mathrm{GeV}$ in low scale supersymmetry when light CP-even Higgs boson is in this the interval $123 \mathrm{GeV} \leq m_{h} \leq 127 \mathrm{GeV}$. This lower bound is very close to the values obtained from fits to the electroweak data [4-7].

In figure 2 we present the results in the $R_{t b \tau}-m_{t}$ and $R_{t b \tau}-m_{h}$ planes. The color coding is the same as in figure 1 . The green points in the $R_{t b \tau}-m_{h}$ plane form a subset of blue points and satisfy the bound $172.3 \mathrm{GeV} \leq m_{t} \leq 174.1 \mathrm{GeV}$.

The horizontal lines in the two planes shown in figure 2 correspond to $5 \% t-b-\tau$ Yukawa coupling unification $\left(R_{t b \tau}=1.05\right)$. From the $R_{t b \tau}-m_{t}$ plane, we see that without requiring any constraints other than REWSB, $t-b-\tau$ Yukawa unification better than $5 \%$ predicts that 

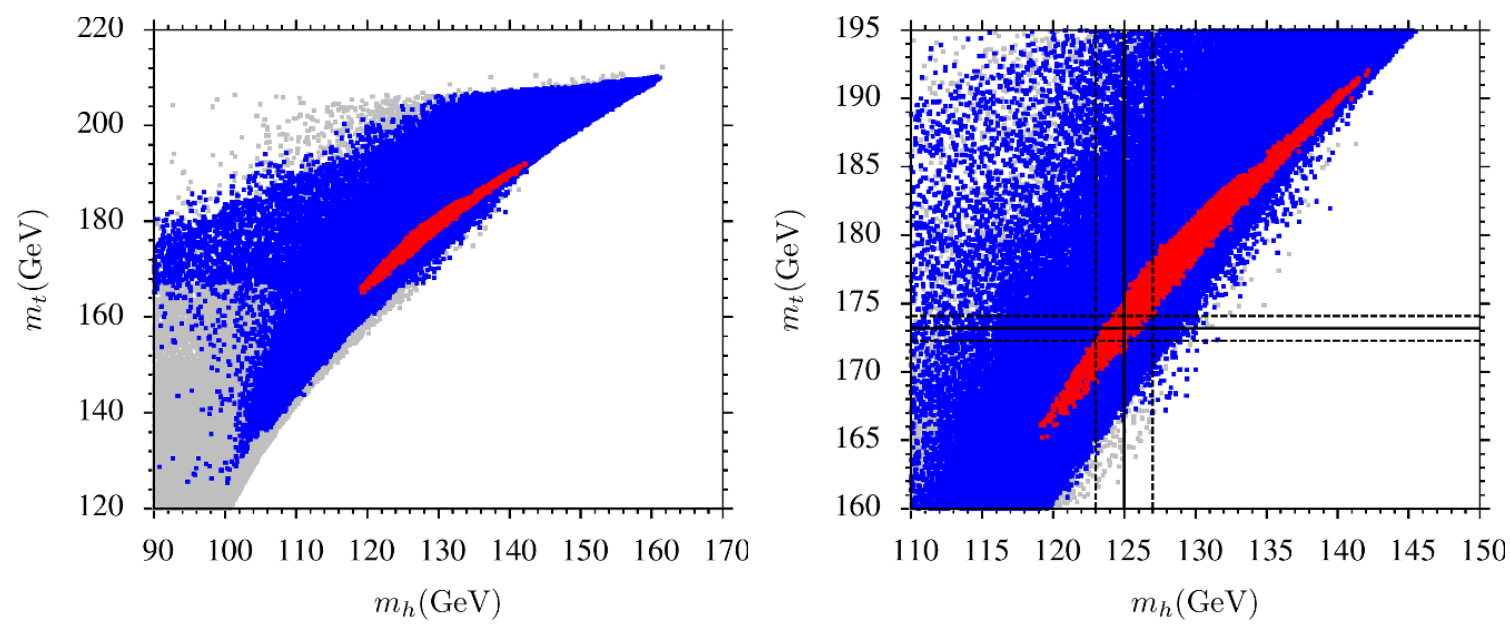

Figure 3. Plots in the $m_{t}-m_{h}$ plane. The right panel is just a zoomed-in version of the left panel. The color coding is the same as in figure 1 with the addition of red points which form a subset of blue points and satisfy $R_{t b \tau}<1.05$.

the top quark cannot be lighter than $140 \mathrm{GeV}$. The top quark mass is further constrained by imposing the phenomenological constraints mentioned in section 2. Even without accounting for the bound on the light $\mathrm{CP}$ even Higgs mass (blue points), the top quark mass is found to lie in the range $166 \mathrm{GeV}<m_{t}<192 \mathrm{GeV}$. While it is not evident from figure 2, of the constraints on the top quark mass implemented in blue, the most severe comes from observation of the decay $B_{s} \rightarrow \mu^{+} \mu^{-}$. It is well known that in low scale supersymmetry models, this flavor-changing decay receives contributions from the exchange of the pseudoscalar Higgs boson $A[61,62]$, and its branching ratio is proportional to $(\tan \beta)^{6} / m_{A}^{4}$. Since $t-b-\tau$ Yukawa unification happens for large $\tan \beta(\approx 47)$ and it prefers relatively small values for the $\mathrm{CP}$ odd pseudoscalar mass $\left(m_{A}<2 \mathrm{TeV}\right)$ in this model $[17,18]$, the top quark mass is severely constrained by the $B_{s} \rightarrow \mu^{+} \mu^{-}$decay. Applying the light CP even Higgs mass bound, the top quark is predicted to have a mass in the narrow window $170 \mathrm{GeV} \leq m_{t} \leq 178 \mathrm{GeV}$.

Next let us consider the constraints on the Higgs boson mass. From the $R_{t b \tau}-m_{h}$ plane, taking into account the collider and B-physics bounds (but excluding the top quark mass bound), $t-b-\tau$ YU better than $5 \%$ requires that the light CP-even Higgs boson mass $m_{h}>119 \mathrm{GeV}$. After imposing the $1 \sigma$ top quark mass bound, the model predicts that the Higgs mass lies in the range $122 \mathrm{GeV} \leq m_{h} \leq 126 \mathrm{GeV}$, in good agreement with the current experimental observations.

We display the correlation between the top quark and Higgs boson masses in the presence of Yukawa unification, in the $m_{t}-m_{h}$ plane in figure 3. The right panel is a zoomed-in version of the left panel in this figure. The color coding is the same as in figure 1, with the addition of red points which form a subset of blue points and satisfy $R_{t b \tau}<1.05$.

The sharp edge towards the right in the $m_{t}-m_{h}$ plane shows that one requires a heavy top quark in order to obtain a Higgs boson mass of $125 \mathrm{GeV}$. We also observe that requiring $5 \%$ or better Yukawa unification makes the Higgs and top quark masses strongly correlated. 
Requiring Yukawa unification along with a $125 \mathrm{GeV}$ Higgs boson mass, one predicts the top quark mass to be in the interval $172 \mathrm{GeV} \leq m_{t} \leq 175 \mathrm{GeV}$. Conversely, requiring Yukawa unification along with the top quark mass to be at its experimentally observed central value, the Higgs boson mass is predicted to be in the range $124 \mathrm{GeV} \leq m_{h} \leq 126 \mathrm{GeV}$.

While there is a few $\mathrm{GeV}$ theoretical error in the calculation of the Higgs mass [63], the strong correlation between the Higgs boson mass, the top quark mass and Yukawa unification seems to be quite compelling in this class of models.

\section{$4 \mathrm{SO}(10)$ GUT with universal gauginos masses}

In this section we consider $t-b$ - $\tau$ Yukawa unification in a supersymmetric $\mathrm{SO}(10)$ GUT model with universal SSB gauginos masses and "Just so" Higgs mass splitting [29, 30]. This GUT scale boundary condition is commonly known as the non-universal Higgs mass (NUHM2) model. The SSB parameters include $m_{16}, M_{1 / 2}, m_{H_{d}}, m_{H_{u}}, A_{0}$, $\tan \beta$. This model predicts a heavy sfermion spectrum $\left(m_{16} \gtrsim 20 \mathrm{TeV}\right)$ but relatively light gaugino masses [60]. For instance, the gluinos in this scenario are not heavier than $3 \mathrm{TeV}$ or so [64], which can be tested at the LHC. We next investigate the allowed range for the top quark and Higgs boson masses in this scenario.

We have performed random scans for the following ranges of parameters:

$$
\begin{aligned}
0 & \leq m_{16} \leq 21 \mathrm{TeV} \\
0 & \leq M_{1 / 2} \leq 5 \mathrm{TeV} \\
0 & \leq m_{H_{d}} \leq 27 \mathrm{TeV} \\
0 & \leq m_{H_{u}} \leq 25 \mathrm{TeV} \\
-36 & \leq A_{0} / m_{16} \leq 3 \mathrm{TeV} \\
1.1 & \leq \tan \beta \leq 60 \\
0 & \leq m_{t} \leq 220 \mathrm{GeV} \\
\mu & >0 .
\end{aligned}
$$

In figure 4 , we present results in the $m_{t}-\tan \beta$ and $m_{t}-M_{S}$ planes. The color coding is the same as in figure 1

In contrast to the $\mathrm{SO}(10)$ model discussed in section 3 , one can see from the $m_{t}$ - $\tan \beta$ plane that there is no restriction in this version of $\mathrm{SO}(10)$ on either the top quark mass or the value of $\tan \beta$ from the point of view of REWSB. After applying sparticle mass bounds and constraints from B-physics, the lower bound on the top quark mass is dramatically increased (blue points in figure 4). The lower bound for blue points in figure 4 appears to be higher compared to the bounds obtained from figure 1. However, this is likely due to a lack of statistics, as indicated in the $m_{t}-M_{S}$ plane, figure 4 . Indeed, we have collected far less data in this case compared to the model presented in section 3. A more exhaustive study will likely fill the regions around the isolated blue points in $m_{t}$-tan $\beta$ plane. The main reason for a lack of extensive statistics for this model is that we are mainly interested in the correlation between the top quark and Higgs boson masses, and this is amply illustrated by 

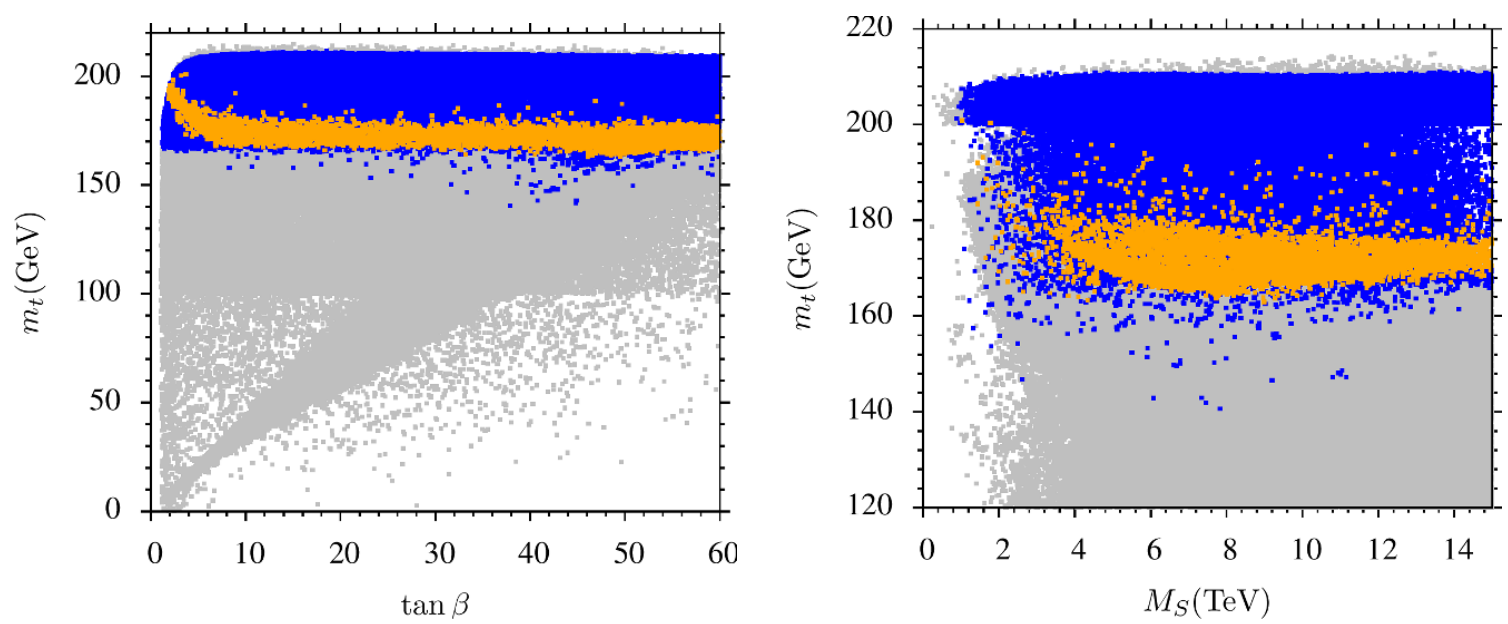

Figure 4. Plots in the $m_{t}$-tan $\beta$ and $m_{t}-M_{S}$ planes. Color coding same as in figure 1.
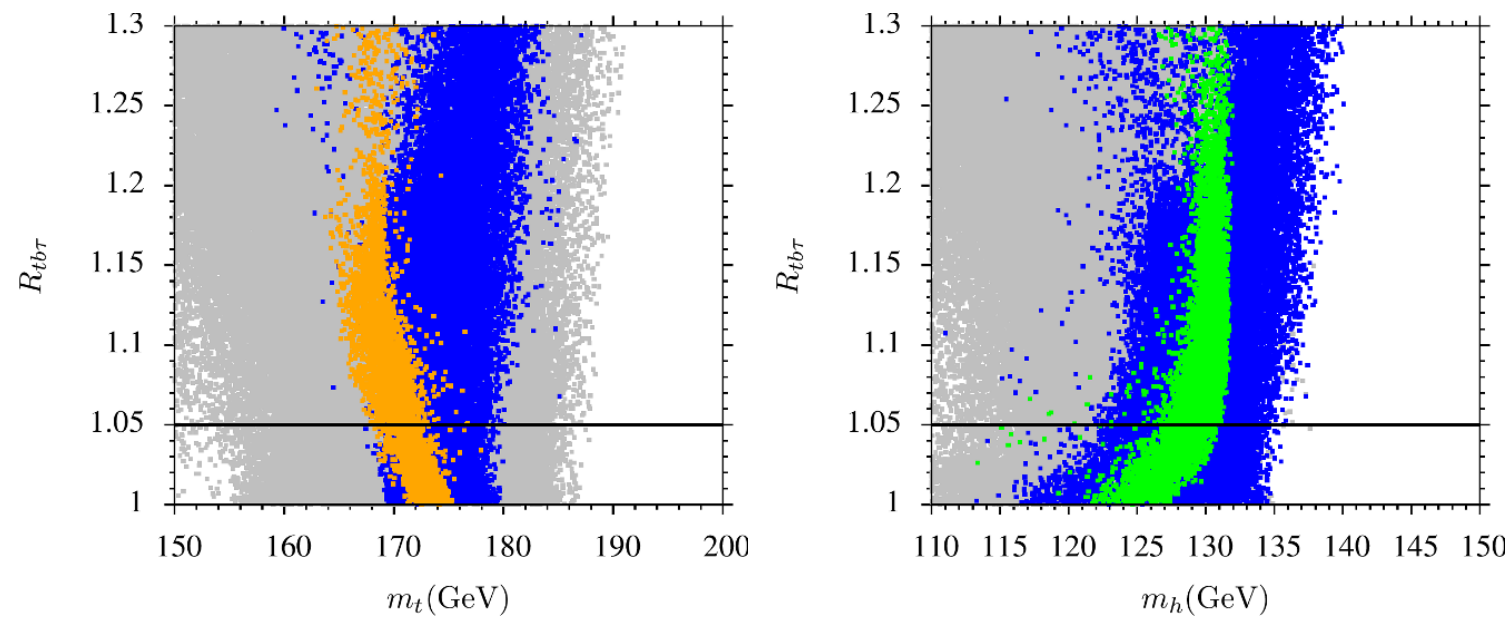

Figure 5. Plots in $R_{t b \tau^{-}} m_{t}$ and $R_{t b \tau}-m_{h}$ planes. The color coding is the same as figure 2. Green points in the $R_{t b \tau}-m_{t}$ plane belong to a subset of blue points and satisfy the top quark mass bound $172.3 \mathrm{GeV} \leq m_{t} \leq 174.1 \mathrm{GeV}$.

the data that we have collected as is obvious by focusing on the orange points in figure 4 . As a reminder to the reader, the orange points form a subset of the blue ones and require the Higgs boson to have a mass in the range $123 \mathrm{GeV} \leq m_{h} \leq 127 \mathrm{GeV}$. This constraint on $m_{h}$ requires, in turn, that $m_{t}$ is restricted to lie in the range $164 \mathrm{GeV}<m_{t}<200 \mathrm{GeV}$.

In figure 5 we show the results in the $R_{t b \tau}-m_{t}$ and $R_{t b \tau}-m_{h}$ planes. The color coding is the same as in figure 4 . The green points in the $R_{t b \tau}-m_{t}$ plane belong to a subset of blue points and satisfy the $1 \sigma$ top quark mass bound $172.3 \mathrm{GeV} \leq m_{t} \leq 174.1 \mathrm{GeV}$.

As shown in figure 5, requiring REWSB and $t-b-\tau$ Yukawa unification better than $5 \%$ requires that $m_{t}$ is heavier than $140 \mathrm{GeV}$ and lighter than $188 \mathrm{GeV}$. The top quark mass gets confined to the range $166 \mathrm{GeV}<m_{t}<180 \mathrm{GeV}$ after applying the collider and Bphysics constraints on the data from section 2 (excluding the Higgs boson mass bound). This bound is virtually unchanged after applying the experimental bound on the Higgs 

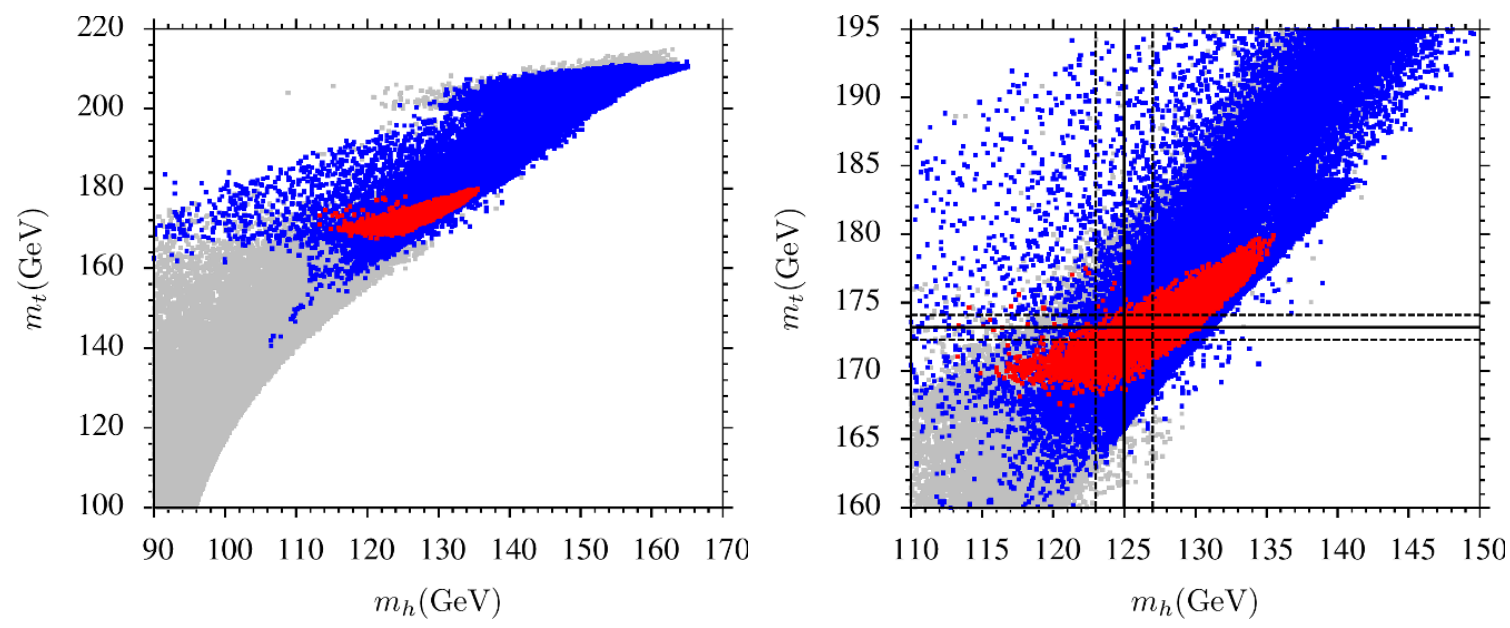

Figure 6. Plots in $m_{t}-m_{h}$ plane. Color coding same as figure 3.

mass (orange points in the figure). The most severe constraint which gives rise to the blue region comes from the decay $b \rightarrow s \gamma$. This is because in low scale supersymmetry the dominant contribution to the $b \rightarrow s \gamma$ branching ratio is proportional to $\mu A_{t} \tan \beta$, while Yukawa unification with universal gaugino masses at $M_{\mathrm{GUT}}$ requires $A_{t} \gtrsim 15 \mathrm{TeV}$ [60]. The SUSY contribution to $B R\left(B_{s} \rightarrow \mu^{+} \mu^{-}\right)$is suppressed in this case because, as pointed out in [64], $m_{A}>3 \mathrm{TeV}$ in this scenario.

The results in the $m_{t}-m_{h}$ plane in figure 6 show the correlation between the top quark and Higgs boson masses in the presence of Yukawa unification. This figure is similar in spirit to figure 3 and shares its color coding.

The sharp right edge in the $m_{t}-m_{h}$ plane is retained in this version of the $\mathrm{SO}(10)$ model, and it shows that one needs a heavy top quark in order to obtain a $125 \mathrm{GeV}$ Higgs boson. We also note that $5 \%$ or better Yukawa unification (red points) makes the Higgs and top quark mass interdependence stronger as in the $\mathrm{SO}(10)$ model of section 3. Requiring the Higgs boson to have a mass of $125 \mathrm{GeV}$ yields a top quark mass in the interval $168 \mathrm{GeV} \leq m_{t} \leq 177 \mathrm{GeV}$. However, fixing the top quark mass in this case does not yield a sharp prediction for the Higgs boson mass. For instance, for the measured central value of $m_{t}$, the Higgs boson mass lies in the range $113 \mathrm{GeV} \leq m_{h} \leq 131 \mathrm{GeV}$.

It is interesting to consider $b$ - $\tau$ Yukawa unification which may be more natural with non-universal SSB mass terms in the Higgs sector. In figure 7 we present results in the $m_{t^{-}}$ $m_{h}$ plane with brown points signifying $5 \%$ or better $b-\tau$ Yukawa unification. The rest of the color coding is the same as in figure 1 . We can see two distinct brown regions corresponding to $b$ - $\tau$ Yukawa unification in this figure. The island-like region is nothing but a somewhat larger region than the red region of figure 6. It is larger because $b-\tau$ Yukawa unification is less restrictive than $t-b-\tau$ Yukawa unification. Unlike $t-b-\tau$ Yukawa unification, $b-\tau$ Yukawa unification is realized for both large and small $\tan \beta$ values. The region formed by the isolated brown points in figure 7 corresponds to the case of small $\tan \beta$. As pointed out in [65-67], $b-\tau$ Yukawa unification for small $\tan \beta$ values requires that the sfermion SSB mass terms are larger than $5 \mathrm{TeV}$ or so. Moreover, the non-universal SSB sfermion mass term terms at $M_{\mathrm{GUT}}$ are need to be based on their $\mathrm{SU}(5)$ representation. While we do 


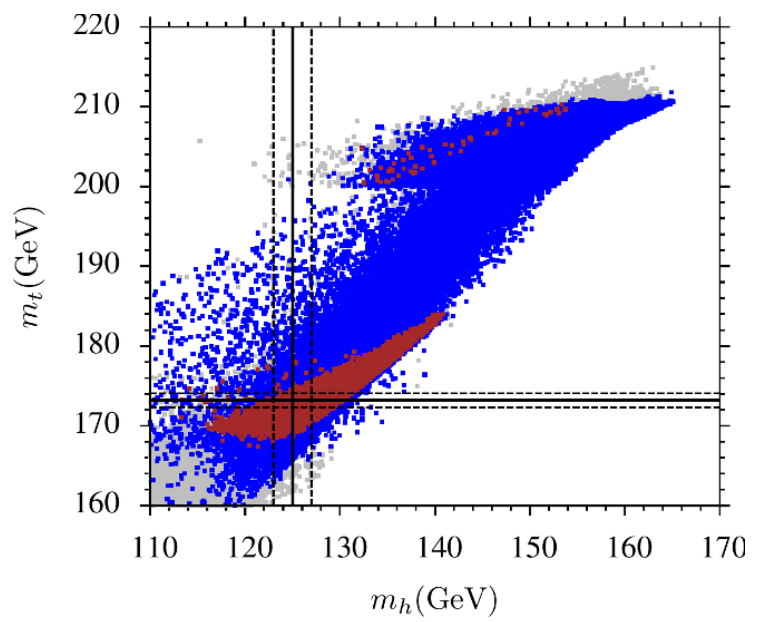

Figure 7. Plots in $m_{t}-m_{h}$ plane. The color coding is the same as figure 1. The brown points form a subset of blue points and represent $5 \%$ or better $b-\tau$ Yukawa unification.

not have non-universal SSB mass terms characteristic of SU(5), the top quark mass is free in our analysis. As a result, $b-\tau$ Yukawa unification does occur for small $\tan \beta$ values, but it occurs in an experimentally unacceptable region for the top quark mass, namely $200-210 \mathrm{GeV}$.

\section{Conclusions}

We have tried to understand the top quark mass from the requirement that $t-b-\tau$ Yukawa coupling unification occurs at $M_{\mathrm{GUT}}$. For this, we consider two $\mathrm{SO}(10)$ GUT models, with one model having universal SSB gaugino masses but non-universal SSB Higgs mass terms. In the second example we have universal Higgs SSB mass terms but non-universal SSB gaugino masses. We have also considered the correlation between the Higgs boson and top quark masses. The upper bound $m_{t} \sim 210 \mathrm{GeV}$ or so on the top quark mass comes from requiring perturbativity of the top quark Yukawa coupling up to scale $M_{\mathrm{GUT}}$. Radiative electroweak symmetry breaking imposes a lower bound on the top quark mass, which depends on $\tan \beta$. In the model with non-universal SSB gaugino masses, applying all the collider and B-physics constraints including the bound on the Higgs boson mass yields the interval $164 \mathrm{GeV} \lesssim m_{t} \lesssim 205 \mathrm{GeV}$. Further, imposing Yukawa coupling unification narrows this mass range, namely $172 \mathrm{GeV} \lesssim m_{t} \lesssim 175 \mathrm{GeV}$.

For the model with non-universal SSB Higgs mass terms, imposing the collider bounds including the bound from the Higgs boson mass, one arrives at a similar range for the top quark mass as the previously quoted result. However, the allowed top quark mass range after imposing Yukawa coupling unification is somewhat more relaxed than in the previous case, to wit $168 \mathrm{GeV} \lesssim m_{t} \lesssim 177 \mathrm{GeV}$, which is quite consistent with the experimental data. Furthermore requiring $b-\tau$ instead of $t-b-\tau$ Yukawa unification in this model relaxes the top quark range, $167 \mathrm{GeV} \lesssim m_{t} \lesssim 182 \mathrm{GeV}$, for large $\tan \beta$ values. The small $\tan \beta$ scenario in this model predicts a top quark mass in the neighborhood of $200 \mathrm{GeV}$ or so, and is, therefore, disfavored. 
The correlation between the Higgs boson mass and the top quark mass is also very interesting, particularly in the model with non-universal SSB gaugino mass terms. By imposing $t-b-\tau$ Yukawa coupling unification and requiring the top quark mass to be close to its observed central value, the Higgs boson mass is founded to lie in the range $124 \mathrm{GeV} \lesssim$ $m_{h} \lesssim 126 \mathrm{GeV}$. This correlation between $m_{t}$ and $m_{h}$ from Yukawa unification is not as strong in the model with non-universal SSB Higgs mass terms. In this case we obtain the result $113 \mathrm{GeV} \lesssim m_{h} \lesssim 131 \mathrm{GeV}$.

\section{Acknowledgments}

We would like to thank K.S. Babu, David Shih, Ayres Freitas, Wang Kai and Tariq Saeed for useful discussions. This work is supported in part by the DOE Grant No. DE-FG0212ER41808 This work used the Extreme Science and Engineering Discovery Environment (XSEDE), which is supported by the National Science Foundation grant number OCI1053575. We also benefitted from the High Performance Computing facilities at the School of Electrical Engineering and Computer Sciences (SEECS) of the National University of Sciences and Technology (NUST), Pakistan. I.G. acknowledges support from the Rustaveli National Science Foundation No. 31/98.

Open Access. This article is distributed under the terms of the Creative Commons Attribution License (CC-BY 4.0), which permits any use, distribution and reproduction in any medium, provided the original author(s) and source are credited.

\section{References}

[1] ATLAS collaboration, Observation of a new particle in the search for the Standard Model Higgs boson with the ATLAS detector at the LHC, Phys. Lett. B 716 (2012) 1 [arXiv: 1207.7214] [INSPIRE].

[2] CMS collaboration, Observation of a new boson at a mass of $125 \mathrm{GeV}$ with the CMS experiment at the LHC, Phys. Lett. B 716 (2012) 30 [arXiv:1207.7235] [INSPIRE].

[3] CDF, D0 collaborations, T. Aaltonen et al., Combination of the top-quark mass measurements from the Tevatron collider, Phys. Rev. D 86 (2012) 092003 [arXiv: 1207.1069] [INSPIRE].

[4] M. Baak et al., The Electroweak Fit of the Standard Model after the Discovery of a New Boson at the LHC, Eur. Phys. J. C 72 (2012) 2205 [arXiv: 1209.2716] [INSPIRE].

[5] O. Eberhardt et al., Impact of a Higgs boson at a mass of $126 \mathrm{GeV}$ on the standard model with three and four fermion generations, Phys. Rev. Lett. 109 (2012) 241802 [arXiv: 1209.1101] [INSPIRE].

[6] J. Erler, Tests of the Electroweak Standard Model, J. Phys. Conf. Ser. 485 (2014) 012010 [arXiv: 1209.3324] [INSPIRE].

[7] M. Ciuchini, E. Franco, S. Mishima and L. Silvestrini, Electroweak Precision Observables, New Physics and the Nature of a 126 GeV Higgs Boson, JHEP 08 (2013) 106 [arXiv: 1306.4644] [INSPIRE].

[8] Y. Okada, M. Yamaguchi and T. Yanagida, Upper bound of the lightest Higgs boson mass in the minimal supersymmetric standard model, Prog. Theor. Phys. 85 (1991) 1 [inSPIRE]. 
[9] Y. Okada, M. Yamaguchi and T. Yanagida, Renormalization group analysis on the Higgs mass in the softly broken supersymmetric standard model, Phys. Lett. B 262 (1991) 54 [INSPIRE].

[10] A. Yamada, Radiative corrections to the Higgs masses in the minimal supersymmetric standard model, Phys. Lett. B 263 (1991) 233 [InSPIRE].

[11] J.R. Ellis, G. Ridolfi and F. Zwirner, Radiative corrections to the masses of supersymmetric Higgs bosons, Phys. Lett. B 257 (1991) 83 [INSPIRE].

[12] J.R. Ellis, G. Ridolfi and F. Zwirner, On radiative corrections to supersymmetric Higgs boson masses and their implications for LEP searches, Phys. Lett. B 262 (1991) 477 [InSPIRE].

[13] H.E. Haber and R. Hempfling, Can the mass of the lightest Higgs boson of the minimal supersymmetric model be larger than m(Z)?, Phys. Rev. Lett. 66 (1991) 1815 [INSPIRE].

[14] B. Ananthanarayan, G. Lazarides and Q. Shafi, Top mass prediction from supersymmetric guts, Phys. Rev. D 44 (1991) 1613 [inSPIRE].

[15] S.I. Gogolev et al., Total cross-section of the reaction $\pi^{+} d \rightarrow p p$ at pion energies $26 \mathrm{MeV}$ to $40 \mathrm{MeV}$, Phys. Lett. B 300 (1993) 24 [INSPIRE].

[16] Q. Shafi and B. Ananthanarayan, Will LEP-2 narrowly miss the Weinberg-Salam Higgs boson?, Trieste HEP Cosmol. 1 (1991) 233 [INSPIRE].

[17] I. Gogoladze, Q. Shafi and C.S. Un, Higgs Boson Mass from t-b- $\tau$ Yukawa Unification, JHEP 08 (2012) 028 [arXiv:1112.2206] [INSPIRE].

[18] M. Adeel Ajaib, I. Gogoladze, Q. Shafi and C.S. Un, A Predictive Yukawa Unified SO(10) Model: Higgs and Sparticle Masses, JHEP 07 (2013) 139 [arXiv:1303.6964] [INSPIRE].

[19] I. Gogoladze, R. Khalid and Q. Shafi, Yukawa Unification and Neutralino Dark Matter in $\mathrm{SU}(4)_{c} \times \mathrm{SU}(2)_{L} \times \mathrm{SU}(2)_{R}$, Phys. Rev. D 79 (2009) 115004 [arXiv:0903.5204] [inSPIRE].

[20] I. Gogoladze, R. Khalid, S. Raza and Q. Shafi, $t-b-\tau$ Yukawa unification for $\mu<0$ with a sub-TeV sparticle spectrum, JHEP 12 (2010) 055 [arXiv: 1008.2765] [INSPIRE].

[21] I. Gogoladze, R. Khalid, S. Raza and Q. Shafi, Higgs and Sparticle Spectroscopy with Gauge-Yukawa Unification, JHEP 06 (2011) 117 [arXiv:1102.0013] [INSPIRE].

[22] I. Gogoladze, Q. Shafi and C.S. Un, SO(10) Yukawa Unification with $\mu<0$, Phys. Lett. B 704 (2011) 201 [arXiv:1107.1228] [INSPIRE].

[23] M. Badziak, M. Olechowski and S. Pokorski, Yukawa unification in $\mathrm{SO}(10)$ with light sparticle spectrum, JHEP 08 (2011) 147 [arXiv:1107.2764] [INSPIRE].

[24] M. Badziak, Yukawa unification in SUSY SO(10) in light of the LHC Higgs data, Mod. Phys. Lett. A 27 (2012) 1230020 [arXiv: 1205.6232] [INSPIRE].

[25] A.S. Joshipura and K.M. Patel, Yukawa coupling unification in $\mathrm{SO}(10)$ with positive $\mu$ and a heavier gluino, Phys. Rev. D 86 (2012) 035019 [arXiv:1206.3910] [InSPIRE].

[26] A. Anandakrishnan, S. Raby and A. Wingerter, Yukawa Unification Predictions for the LHC, Phys. Rev. D 87 (2013) 055005 [arXiv: 1212.0542] [INSPIRE].

[27] A. Anandakrishnan and S. Raby, Yukawa Unification Predictions with effective "Mirage" Mediation, Phys. Rev. Lett. 111 (2013) 211801 [arXiv:1303.5125] [INSPIRE].

[28] M. Badziak, M. Olechowski and S. Pokorski, Light staus and enhanced Higgs diphoton rate with non-universal gaugino masses and $\mathrm{SO}(10)$ Yukawa unification, JHEP 10 (2013) 088 [arXiv: 1307.7999] [INSPIRE]. 
[29] T. Blazek, R. Dermisek and S. Raby, Predictions for Higgs and supersymmetry spectra from $\mathrm{SO}(10)$ Yukawa unification with mu greater than 0, Phys. Rev. Lett. 88 (2002) 111804 [hep-ph/0107097] [INSPIRE].

[30] T. Blazek, R. Dermisek and S. Raby, Yukawa unification in SO(10), Phys. Rev. D 65 (2002) 115004 [hep-ph/0201081] [INSPIRE].

[31] F.E. Paige, S.D. Protopopescu, H. Baer and X. Tata, ISAJET 7.69: A Monte Carlo event generator for $p p, \bar{p} p$ and $e^{+} e^{-}$reactions, hep-ph/0312045 [INSPIRE].

[32] J. Hisano, H. Murayama and T. Yanagida, Nucleon decay in the minimal supersymmetric SU(5) grand unification, Nucl. Phys. B 402 (1993) 46 [hep-ph/9207279] [INSPIRE].

[33] Y. Yamada, SUSY and GUT threshold effects in SUSY SU(5) models, Z. Phys. C 60 (1993) 83 [INSPIRE].

[34] J.L. Chkareuli and I.G. Gogoladze, Unification picture in minimal supersymmetric SU(5) model with string remnants, Phys. Rev. D 58 (1998) 055011 [hep-ph/9803335] [INSPIRE].

[35] D.M. Pierce, J.A. Bagger, K.T. Matchev and R.-j. Zhang, Precision corrections in the minimal supersymmetric standard model, Nucl. Phys. B 491 (1997) 3 [hep-ph/9606211] [INSPIRE].

[36] G. Bélanger, F. Boudjema, A. Pukhov and R.K. Singh, Constraining the MSSM with universal gaugino masses and implication for searches at the LHC, JHEP 11 (2009) 026 [arXiv: 0906.5048] [INSPIRE].

[37] L.E. Ibáñez and G.G. Ross, $\mathrm{SU}(2)_{L} \times \mathrm{U}(1)$ Symmetry Breaking as a Radiative Effect of Supersymmetry Breaking in Guts, Phys. Lett. B 110 (1982) 215 [INSPIRE].

[38] K. Inoue, A. Kakuto, H. Komatsu and S. Takeshita, Aspects of Grand Unified Models with Softly Broken Supersymmetry, Prog. Theor. Phys. 68 (1982) 927 [Erratum ibid. 70 (1983) 330] [INSPIRE].

[39] L.E. Ibáñez, Locally Supersymmetric SU(5) Grand Unification, Phys. Lett. B 118 (1982) 73 [INSPIRE].

[40] J.R. Ellis, D.V. Nanopoulos and K. Tamvakis, Grand Unification in Simple Supergravity, Phys. Lett. B 121 (1983) 123 [INSPIRE].

[41] L. Álvarez-Gaumé, J. Polchinski and M.B. Wise, Minimal Low-Energy Supergravity, Nucl. Phys. B 221 (1983) 495 [inSPIRE].

[42] Particle Data Group collaboration, K. Nakamura et al., Review of particle physics, J. Phys. G 37 (2010) 075021 [inSPIRE].

[43] H. Baer, C. Balázs and A. Belyaev, Neutralino relic density in minimal supergravity with coannihilations, JHEP 03 (2002) 042 [hep-ph/0202076] [INSPIRE].

[44] H. Baer, C. Balázs, J. Ferrandis and X. Tata, Impact of muon anomalous magnetic moment on supersymmetric models, Phys. Rev. D 64 (2001) 035004 [hep-ph/0103280] [INSPIRE].

[45] F. Mahmoudi, SuperIso v2.3: A program for calculating flavor physics observables in Supersymmetry, Comput. Phys. Commun. 180 (2009) 1579 [arXiv:0808.3144] [INSPIRE].

[46] CDF collaboration, T. Aaltonen et al., Search for $B_{s}^{0} \rightarrow \mu^{+} \mu^{-}$and $B_{d}^{0} \rightarrow \mu^{+} \mu^{-}$decays with $2 \mathrm{fb}^{-1}$ of pp collisions, Phys. Rev. Lett. 100 (2008) 101802 [arXiv:0712.1708] [INSPIRE].

[47] Heavy Flavor Averaging Group collaboration, E. Barberio et al., Averages of b-hadron and c-hadron Properties at the End of 2007, arXiv:0808.1297 [INSPIRE]. 
[48] Muon G-2 collaboration, G.W. Bennett et al., Final Report of the Muon E821 Anomalous Magnetic Moment Measurement at BNL, Phys. Rev. D 73 (2006) 072003 [hep-ex/0602035] [INSPIRE].

[49] ATLAS collaboration, Search for squarks and gluinos with the ATLAS detector in final states with jets and missing transverse momentum using $4.7 \mathrm{fb}^{-1}$ of $\sqrt{s}=7 \mathrm{TeV}$ proton-proton collision data, Phys. Rev. D 87 (2013) 012008 [arXiv:1208.0949] [INSPIRE].

[50] CMS collaboration, Search for supersymmetry in hadronic final states using MT2 in pp collisions at $\sqrt{s}=7 \mathrm{TeV}$, JHEP 10 (2012) 018 [arXiv:1207.1798] [INSPIRE].

[51] CMS collaboration, Higgs to tau tau (MSSM) (HCP), CMS-PAS-HIG-12-050.

[52] A.H. Chamseddine, R.L. Arnowitt and P. Nath, Locally Supersymmetric Grand Unification, Phys. Rev. Lett. 49 (1982) 970 [InSPIRE].

[53] R. Barbieri, S. Ferrara and C.A. Savoy, Gauge Models with Spontaneously Broken Local Supersymmetry, Phys. Lett. B 119 (1982) 343 [INSPIRE].

[54] N. Ohta, Grand unified theories based on local supersymmetry, Prog. Theor. Phys. 70 (1983) 542 [INSPIRE].

[55] L.J. Hall, J.D. Lykken and S. Weinberg, Supergravity as the Messenger of Supersymmetry Breaking, Phys. Rev. D 27 (1983) 2359 [INSPIRE].

[56] L.J. Hall, R. Rattazzi and U. Sarid, The top quark mass in supersymmetric $\mathrm{SO}(10)$ unification, Phys. Rev. D 50 (1994) 7048 [hep-ph/9306309] [INSPIRE].

[57] B. Ananthanarayan and P.N. Pandita, Sparticle Mass Spectrum in Grand Unified Theories, Int. J. Mod. Phys. A 22 (2007) 3229 [arXiv:0706.2560] [InSPIRE].

[58] S. Bhattacharya, A. Datta and B. Mukhopadhyaya, Non-universal gaugino masses: a signal-based analysis for the Large Hadron Collider, JHEP 10 (2007) 080 [arXiv:0708.2427] [INSPIRE].

[59] S.P. Martin, Non-universal gaugino masses from non-singlet F-terms in non-minimal unified models, Phys. Rev. D 79 (2009) 095019 [arXiv:0903.3568] [InSPIRE].

[60] H. Baer, S. Kraml, S. Sekmen and H. Summy, Dark matter allowed scenarios for Yukawa-unified SO(10) SUSY GUTs, JHEP 03 (2008) 056 [arXiv:0801.1831] [INSPIRE].

[61] S.R. Choudhury and N. Gaur, Dileptonic decay of B(s) meson in SUSY models with large tan Beta, Phys. Lett. B 451 (1999) 86 [hep-ph/9810307] [INSPIRE].

[62] K.S. Babu and C.F. Kolda, Higgs mediated $B^{0} \rightarrow \mu^{+} \mu^{-}$in minimal supersymmetry, Phys. Rev. Lett. 84 (2000) 228 [hep-ph/9909476] [INSPIRE].

[63] P. Draper, G. Lee and C.E.M. Wagner, Precise Estimates of the Higgs Mass in Heavy SUSY, Phys. Rev. D 89 (2014) 055023 [arXiv: 1312.5743] [INSPIRE].

[64] H. Baer, S. Raza and Q. Shafi, A heavier gluino from $t-b-\tau$ Yukawa-unified SUSY, Phys. Lett. B 712 (2012) 250 [arXiv: 1201.5668] [INSPIRE].

[65] I. Gogoladze, R. Khalid, N. Okada and Q. Shafi, Soft Probes of SU(5) Unification, Phys. Rev. D 79 (2009) 095022 [arXiv: 0811.1187] [INSPIRE].

[66] I. Gogoladze, S. Raza and Q. Shafi, Light Stop from b-tau Yukawa Unification, Phys. Lett. B 706 (2012) 345 [arXiv:1104.3566] [INSPIRE].

[67] H. Baer, I. Gogoladze, A. Mustafayev, S. Raza and Q. Shafi, Sparticle mass spectra from SU(5) SUSY GUT models with b - $\tau$ Yukawa coupling unification, JHEP 03 (2012) 047 [arXiv: 1201.4412] [INSPIRE]. 